\title{
Analysis of Video Fluoroscopic Swallowing Study in Patients with Vocal Cord Paralysis
}

\author{
Yoon Young Jang, MD, \\ Department of Rehabilitation Medicine, Dankook University College of Medicine, Chugnam, \\ Republic of Korea
}

\begin{abstract}
Sang Jun Lee, MD, PhD, Department of Otolaryngology/Head and Neck Surgery, Dankook University College of Medicine, Chugnam, Republic of Korea
\end{abstract}

Jae Yong Jeon, MD, and Department of Rehabilitation Medicine, Ulsan University College of Medicine Asan Medical Center, Seoul, Republic of Korea

Seong Jae Lee, MD, PhD

Department of Rehabilitation Medicine, Dankook University Hospital, San 16-5, Anseo-dong, Dongnam-gu, Cheonan, Chugnam 330-715, Republic of Korea

Seong Jae Lee: rmlee@dankook.ac.kr

\begin{abstract}
We reviewed the findings of a video fluoroscopic swallowing study (VFSS) of 28 patients with vocal cord paralysis (VCP) who complained of swallowing difficulties. VFSSs were performed with thick and thin liquid using modified Logemann methods. The patients were grouped according to whether their VCP was of central or peripheral origin, and the VFSS findings of the groups were compared. The patients showed oral phase dysfunction and pharyngeal dysfunction, especially when the cause was of central origin. Oral phase abnormalities were found in 13 patients and pharyngeal phase abnormalities were found in all patients, including penetration in 20 patients and aspiration in 14 patients. Improper lip closure (LC) and bolus formation (BF) and a delay in triggering pharyngeal swallow (TPS) and upper esophageal sphincter release (UESR) were significantly more frequent in patients with central VCP. With thin-liquid swallowing, pharyngeal transit time (PTT) and pharyngeal delay time (PDT) were significantly more prolonged in central VCP. The results suggest that the delay in triggering and poor coordination of swallowing were profound in patients with central VCP, but dysfunction in peripheral VCP may originate from poor pharyngeal movement.
\end{abstract}

\section{Keywords}

Vocal cord paralysis; Video fluoroscopic swallowing study; Dysphagia; Deglutition; Deglutition disorders

\footnotetext{
Vocal cord paralysis (VCP) implies vocal fold immobility due to neurologic pathologies
} such as vagus, recurrent laryngeal, and superior laryngeal neuropathy. Central nervous

(C) Springer Science+Business Media, LLC 2011

Correspondence to: Seong Jae Lee, rmlee@dankook . ac . kr. 
system disorders (stroke, brain tumor, and traumatic brain injury) can also cause VCP [1]. VCP may present as dysphonia, hoarseness, breathlessness, and also choking episodes.

The control of the swallowing process represents sensorimotor coordination among cranial nerves, brain stem, and cortical central pathways [2]. The peripheral innervations of the swallowing process are provided by cranial nerves, including trigeminal, facial, glossopharyngeal, hypoglossal nerves [2,3]. The brain stem plays the most important role in the swallowing process [2]. The cranial motor and sensory nuclei (the trigeminal nucleus, the facial nucleus, the nucleus ambiguus, the nucleus tractus solitaries, and the hypoglossal nucleus) located in the brain stem are involved [2]. The face area in primary motor cortex (M1) and the primary sensory cortex (S1) are also involved [2].

Several studies on the swallowing difficulties of patients with VCP have been performed. Previous studies have reported that nearly $56 \%$ of patients with VCP have swallowing dysfunction $[4,5]$. Tabaee et al. [6] suggested that dysphagia in patients with VCP has multifactorial causes, including limited airway protection and decreased laryngopharyngeal sensation based on the findings of flexible endoscopy. Heitmiller et al. [7] asserted that aspiration resulted from impaired airway protection rather than esophageal obstruction in patients with unilateral vocal fold motion impairment, and recommended video pharyngoesophagography as the technique of choice to identify patients at risk for aspiration. In a study by Bhattacharyya et al. [8], pharyngeal phase abnormalities such as delayed initiation of swallowing, reduced laryngeal elevation, and reduced upper esophageal sphincter (UES) opening were found in video fluoroscopic swallowing studies (VFSS). In a manometric evaluation by Wilson et al. [5], patients with central- and peripheral-type VCP had different findings during the swallowing process, suggesting that the location of pathologic lesions is one of the important factors of swallowing dysfunction.

Although VFSS is recognized as a valuable technique in the evaluation of patients with swallowing difficulties, including VCP, a comprehensive evaluation of VFSS findings of patients with VCP has not been attempted. Moreover, the difference in VFSS findings between peripheral- and central-type VCP has not been investigated. Therefore, we conducted this study to determine the characteristics of VFSS findings in patients with VCP. The VFSS findings of peripheral- and central-type VCP were also compared.

\section{Subjects and Methods}

\section{Subjects}

After obtaining approval from the Institutional Review Board of Dankook University Hospital, the subjects were identified from the database of the Department of Rehabilitation Medicine. Patients who were diagnosed with VCP from those who were evaluated by VFSS because of swallowing difficulties or aspiration symptoms were included in the study. Documentation of VCP mandated a visual inspection of the patient's vocal cords by an otolaryngologist in our institution to confirm the diagnosis. The medical records were reviewed to determine age, gender, documentation of VCP, cause of VCP, and aspiration symptoms. We excluded patients with structural abnormalities of the oral cavity, pharynx, or larynx.

Twenty-eight patients were identified and included in the analysis. All subjects were adults, with a mean age of $56.2 \pm 16.6$ years. Eighteen patients (11 males and 7 females) who were diagnosed with VCP of peripheral origin were designated as the peripheral VCP group, and ten patients ( 3 males and 7 females) who were diagnosed with VCP of central origin were designated as the central VCP group. The causes of peripheral VCP included recurrent laryngeal nerve injuries $(n=2)$, vagus nerve injuries $(n=2)$, and idiopathic VCP $(n=14)$. 
The central VCP group consisted of seven patients with strokes ( 6 patients with brain stem infarction and 1 with cortical stroke), two patients with traumatic brain injuries, and one patient with a brain tumor. Every effort was made to rule out peripheral causes in the central $\mathrm{VCP}$, but there still remains a possibility of coexistence.

The mean interval from onset of VCP to the time of the VFSS was $49.0 \pm 62.2$ days, and there was no significant difference between the central VCP group (43.7 \pm 66.8 days) and peripherals VCP group $(58.6 \pm 55.2$ days $)$.

\section{Methods}

With the subject seated, VFSS was performed using modified Logemann methods [3]. The swallowing images in the lateral projections were recorded and stored digitally at a speed of 30 frames per second. First, a screening test was done using $1 \mathrm{ml}$ of thin liquid. After that, a 3-ml thick liquid swallowing test and a 3-ml thin-liquid swallowing test were done in sequence. Each subject completed four or five swallows of thick (200\% diluted barium) and thin liquids (70\% diluted barium).

The images of the VFSS records were analyzed frame by frame by the same physiatrist with expertise in VFSS analysis. The swallowing processes in the oral and pharyngeal phases were evaluated, as shown in Table 1. In the oral phase, lip closure (LC), i.e., maintenance of labial seal to ensure that no food or liquid falls from the mouth [3]; bolus formation (BF), i.e., mastication and preparation of a semicohesive bolus or ball (3); and residue of the oral cavity (ROC), i.e., residue of the barium on the floor of the mouth, tongue, hard palate, and anterolateral sulcus (3) were assessed, and the oral transit time (OTT) was measured. In the pharyngeal phase, triggering of pharyngeal swallow (TPS), i.e., elevation and anterior movement of the hyoid and larynx with the bolus head passing the spot where the lower edge of the mandible crosses the tongue base [3]; laryngeal elevation and epiglottic closure (LEEC), i.e., getting closer and tilting forward of the arytenoids to the base of the epiglottis and closing the airway as the larynx is elevated [3]; residue in the valleculae (RV) and pyriform sinus (RP), i.e., barium residue on the vallecula and pyriform sinus after swallowing [3]; and upper esophageal sphincter release (UESR), i.e., opening of the UES with the bolus head reaching the cricopharyngeal sphincter [3] were assessed, and the pharyngeal transit time (PTT) and pharyngeal delay time (PDT) were measured.

The OTT was defined as the length of time from the first frame showing backward movement of food to when the bolus head reaches the spot where the lower edge of the mandible crosses the tongue base [3]. The PTT was defined as the time from the point at which the bolus head passes the spot where the lower edge of the mandible crosses the tongue base to when the bolus tail passes through the cricopharyngeal region, which is located behind the cartilage, approximately $1 \mathrm{~cm}$ below the true vocal folds [3]. The PDT is a component of the PTT and was defined as the time from the arrival of the bolus at the point where the lower edge of the mandible crosses the tongue base until the pharyngeal swallow is triggered [3]. Triggering or onset of the pharyngeal swallow was defined as the first video frame that showed laryngeal elevation as a part of the pharyngeal swallowing complex [3].

The presence of penetration or aspiration was also noted. Penetration was defined as the presence of the bolus within the laryngeal inlet, but above the true vocal cord [3]. Aspiration was defined as the passage of the bolus material beyond the level of the true vocal cord [3]. The parameters mentioned above were determined based on previous studies of VFSS [9$11]$. 


\section{Statistical Analysis}

The frequency of abnormalities of the peripheral and central VCP groups was compared using Fisher's exact test, and the difference in time parameters such as PDT and PTT was tested using the Mann-Whitney test. The statistical significance was set at $P<0.05$.

\section{Results}

\section{Abnormal Findings in VFSS}

Abnormalities in the oral and pharyngeal phases are summarized in Table 2. In the oral phase, $\mathrm{LC}$ was incomplete in 6 patients $(21.4 \%)$, BF was abnormal in 9 patients $(32.1 \%)$, and excessive residue remained in the oral cavity after deglutition in 7 patients $(25 \%)$. The OTT was delayed in 7 patients (25\%).

In the pharyngeal phase, delayed TPS, excessive RP, and prolonged PTT with swallowing of thick liquid showed relatively high occurrence $(>60 \%)$, and incomplete LEEC and excessive RV showed very high occurrence (75\%). Aspiration was documented in 14 patients (50\%), and 20 patients $(71.4 \%)$ showed penetration. In general, swallowing defects were more frequent in the pharyngeal phase than the oral phase.

\section{Peripheral versus Central VCP}

In the oral phase, the frequency of abnormalities was higher in the central VCP group (40$60 \%$ ) than in the peripheral VCP group (0-16.7\%; Table 3). Incomplete LC and BF occurred more frequently in the central VCP group than in the peripheral VCP group, and the difference was statistically significant $(P<0.05$; Table 3$)$. However, the frequencies of delayed OTT and excessive RO were not significantly different.

In the pharyngeal phase, the central VCP group had a higher frequency of abnormalities throughout the parameters (60-90\%; Table 4). The differences in the frequency of TPS, UESR, and aspiration were statistically significant $(P<0.05$; Table 4$)$. The peripheral VCP group had a higher frequency of abnormalities in LEEC (66.7\%), RV (72.2\%), and RP $(55.6 \%)$ than other parameters, but the frequencies were still lower than those of the central VCP group.

Among the time parameters, PTT and PDT with thin liquids were significantly longer in the central VCP group $(4.5 \pm 4.9 \mathrm{~s}$ and $2.0 \pm 2.4 \mathrm{~s}$, respectively) than in the peripheral VCP group $(0.9 \pm 0.3 \mathrm{~s}$ and $0.4 \pm 0.2 \mathrm{~s}$, respectively; $P<0.05$; Fig. 1$)$.

\section{Discussion}

Most studies of patients with VCP have concluded that the main cause of dysphagia is the reduced mechanical protection of the glottis [4-6]. However, Tabaee et al. [6] reported that pharyngeal sensory impairment is involved, and Battacharyya et al. [8] suggested that diminished cough strength is also an important cause. The results of the present study suggest that the cause of dysphagia in patients with VCP is a combination of reduced pharyngeal motility, poor coordination of deglutition, and a lack of mechanical protection.

The oral phase abnormalities in patients with VCP have not been addressed in the extant literature. In the current study, oral phase abnormalities were documented in 13 patients (46.4\%). In the central VCP group, oral dysfunction existed in 7 patients (70\%), and only 6 patients $(33.3 \%)$ had oral dysfunction in the peripheral VCP group. Delayed oral transit time tended to occur more frequently in the central VCP group, although this difference did not reach statistical significance $(P=0.063)$. We believe that these results might achieve significance in a larger sample of patients. The oral phase abnormalities in the central VCP 
group may result from incoordination of the swallowing process. However, we cannot completely rule out the possibility of overestimation in the peripheral VCP group because two patients had painful varicella zoster in the perioral area and one patient had idiopathic facial spasms, which may affect the oral motor function.

Heitmiller et al. [7] reported that 38 and $12 \%$ of the patients with unilateral vocal cord immobilization had penetration and aspiration, respectively. Battacharyya et al. [8] reported that the incidences of penetration and aspiration were 31.3 and $23.4 \%$, respectively, in patients with the same vocal cord pathology. In the current study, the incidences of penetration and aspiration were higher than in previous reports. We speculate that the higher incidences of penetration and aspiration can be explained by the difference in the composition of study group. In the current study, the patients with VCP of central origin had higher incidences of penetration and aspiration; in previous studies only a small number of those subjects were included or were not included at all.

In the present study, abnormal findings of VFSS in patients with VCP were shown throughout the oral and pharyngeal phases, and the frequencies of penetration and aspiration were relatively high. Compared with the central VCP group, VFSS abnormalities in the peripheral VCP group were primarily in the pharyngeal phase. Abnormalities of upper esophageal sphincter release, triggering of pharyngeal swallow, and aspiration were significantly more common in the central VCP group. In the peripheral VCP group, abnormalities of laryngeal elevation and epiglottic closure and residue in the valleculae and pyriform sinus were relatively more frequent than abnormalities of other parameters. These results suggest that the major cause of dysphagia in the peripheral VCP group could be reduced pharyngeal movement. On the other hand, a delay in the triggering of the swallowing reflex and poor coordination of the swallowing process could be the main causes of dysphagia in the central VCP group. In view of treatment planning, the results of the present study suggest that the treatment of patients with peripheral VCP needs to be focused on pharyngeal muscle strengthening, but in the patients with central VCP, the treatment should be focused on training to improve triggering and coordination of swallowing.

Waryngeal delay time were significantly longer in the central VCP group, but only with thin liquids. It is known that the pressure generated by the oropharyngeal structures increases with increased bolus viscosity, and the oropharyngeal muscular activity also increases [1214]. It is possible that the oropharyngeal muscular activity was not provoked by the low viscosity of thin liquids.

In this study, male patients made up about $61 \%$ of the peripheral VCP group, whereas females were predominant in the central VCP group. The gender distribution in the peripheral VCP is reasonable, according to previous reports. Klechner et al. [15] reported that males slightly exceeded females (55.6 vs. 44.4\%) in retrospective research of 117 subjects with VCP. Also, males accounted for nearly $66 \%$ in the study by Heitmiller et al. [7]. It is hard to explain the predominance of females in the central VCP group, but we postulate that the distribution was skewed coincidently by the small sample size.

The present study has a few limitations. As mentioned before, the sample size was small. We could not completely rule out the peripheral lesion in central VCP, while we were able to rule out a central lesion in peripheral VCP by means of brain MRI. We could not find evidence in the medical records of the patients with central VCP that suggested peripheral VCP. The subjects had a common manifestation of vocal cord palsy but the specific causes were heterogeneous. Therefore, the high frequency and wide distribution of abnormalities in VFSS, especially in central VCP group, may originate from the differences of the natural course and clinical characteristics among subgroups. Such heterogeneity may limit 
generalization of the results of the present study, which seems to be a common limitation of most studies regarding vocal cord palsy. Stratification of subjects based on etiology was not possible because of the small sample size and retrospective nature of our study, but the present study was not focused on a specific etiology of vocal cord palsy. In future studies, correction and analysis of the data according to etiologic subgroups may be required. Another limitation of the present study is wide range of time from onset of VCP to VFSS. Patients with VCP of different severity and time course could be mixed together, resulting in a wide range of pharyngeal delay time and pharyngeal transit time. Differences in pharyngeal delay time and pharyngeal transit time with thick-liquid swallowing between groups might be masked by the wide range of data.

Nevertheless, this is the first study to evaluate the comprehensive characteristics of VFSS in patients with dysphagia resulting from VCP, and to compare the swallowing patterns of peripheral and central VCP patients using VFSS. In the future, prospective studies are required to establish more accurate diagnosis and avoid overlapping of groups. Follow-up VFSS will be helpful in investigating the clinical course and prognosis of swallowing difficulties of VCP patients. Finally, a study using larger patient group with homogeneous etiology is required to enhance the statistical significance and further elucidate the usefulness of VFSS in vocal cord disorders.

\section{Conclusions}

Patients with VCP and dysphagia can have oral phase dysfunction as well as pharyngeal dysfunction, especially when the cause is of central origin. Abnormal findings of VFSS in patients with VCP frequently occurred in the pharyngeal phase, in which delayed TPS, reduced LEEC, increased RV and RP, and prolonged PTT were particularly common. The main dysfunction in $\mathrm{VCP}$ of central origin could be the delay in triggering and poor coordination of swallowing, but the major problem in VCP of peripheral origin could be impaired pharyngeal movement.

\section{Acknowledgments}

The present research was conducted by the Research Fund of Dankook University in 2009.

\section{References}

1. Rubin AD, Sataloff RT. Vocal fold paresis and paralysis. Otolaryngol Clin North Am. 2007; 40:1109-31. viii-ix. [PubMed: 17765698]

2. Miller AJ. The neurobiology of swallowing and dysphagia. Dev Disabil Res Rev. 2008; 14:77-86. [PubMed: 18646019]

3. Logemann, JA. Evaluation and Treatment of Swallowing Disorders. 2nd. San Diego: College Hill Press; 1998.

4. Ollivere B, Duce K, Rowlands G, Harrison P, O'Reilly BJ. Swallowing dysfunction in patients with unilateral vocal fold paralysis: aetiology and outcomes. J Laryngol Otol. 2006; 120:38-41.

[PubMed: 16359143]

5. Wilson JA, Pryde A, White A, Maher L, Maran AG. Swallowing performance in patients with vocal fold motion impairment. Dysphagia. 1995; 10:149-54. [PubMed: 7614853]

6. Tabaee A, Murry T, Zschommler A, Desloge RB. Flexible endoscopic evaluation of swallowing with sensory testing in patients with unilateral vocal fold immobility: incidence and pathophysiology of aspiration. Laryngoscope. 2005; 115:565-9. [PubMed: 15805859]

7. Heitmiller RF, Tseng E, Jones B. Prevalence of aspiration and laryngeal penetration in patients with unilateral vocal fold motion impairment. Dysphagia. 2000; 15:184-7. [PubMed: 11014880] 
8. Bhattacharyya N, Kotz T, Shapiro J. Dysphagia and aspiration with unilateral vocal cord immobility: incidence, characterization, and response to surgical treatment. Ann Otol Rhinol Laryngol. 2002; 111:672-9. [PubMed: 12184586]

9. Han TR, Paik NJ, Park JW. The functional dysphagia scale using videofluoroscopic swallowing study in stroke patients. J Kor Acad Rehabil Med. 1999; 23:1118-26.

10. Park NJ, Kim IS, Kim JH, Oh BM, Han TR. Clinical validity of the functional dysphagia scale based on videofluoroscopic swallowing study. J Kor Acad Rehabil Med. 2005; 29:43-9.

11. Han TR, Paik NJ, Park JW. Quantifying swallowing function after stroke: a functional dysphagia scale based on videofluoroscopic studies. Arch Phys Med Rehabil. 2001; 82:677-82. [PubMed: 11346847]

12. Perlman AL, Schultz JG, VanDaele DJ. Effects of age, gender, bolus volume, and bolus viscosity on oropharyngeal pressure during swallowing. J Appl Physiol. 1993; 75:33-7. [PubMed: 8376283]

13. Bhattacharyya N, Kotz T, Shapiro J. The effect of bolus consistency on dysphagia in unilateral vocal cord paralysis. Otolaryngol Head Neck Surg. 2003; 129:632-6. [PubMed: 14663428]

14. Dantas RO, Kern MK, Massey BT, Dodds WJ, Kahrilas PJ, Brasseur JG, Cook IJ, Lang IM. Effect of swallowed bolus variables on oral and pharyngeal phases of swallowing. Am J Physiol. 1990; 258:G675-81. [PubMed: 2333995]

15. Kelchner LN, Stemple JC, Gerdeman E, Le Borgne W, Adam S. Etiology, pathophysiology, treatment choices, and voice results for unilateral adductor vocal fold paralysis: a 3-year retrospective. J Voice. 1999; 13:592-601. [PubMed: 10622524] 


\section{Time (sec)}

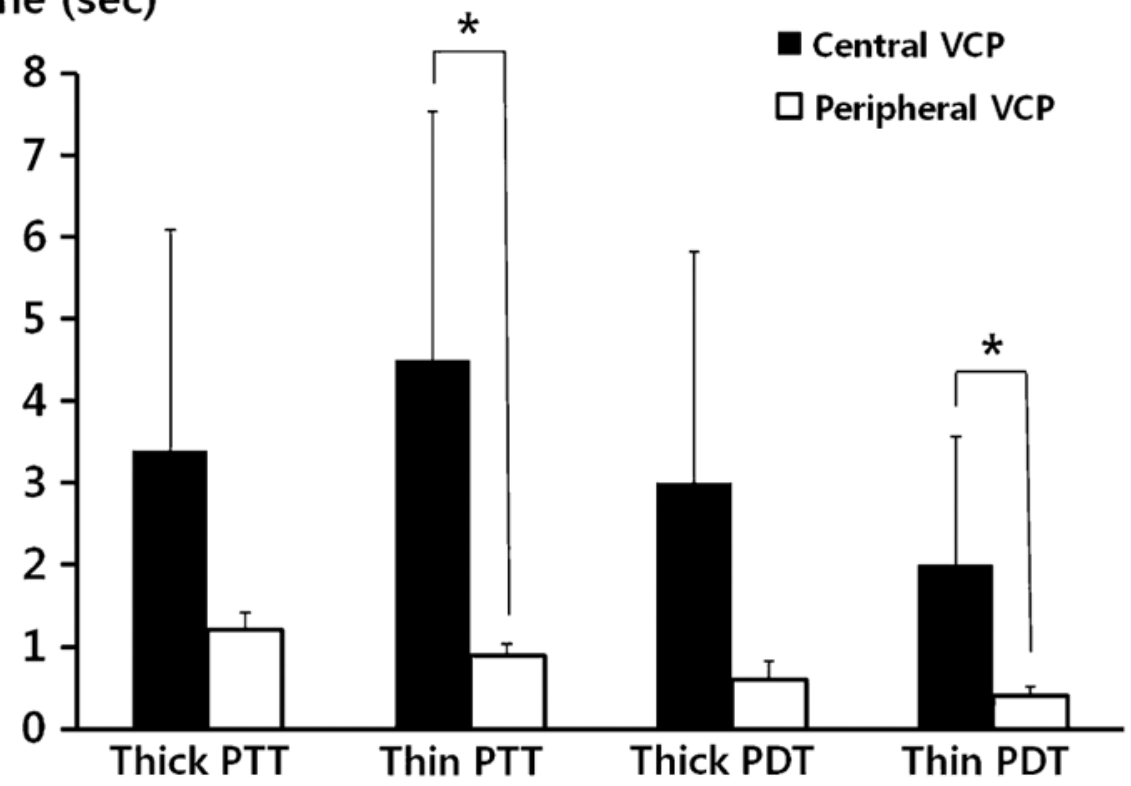

Fig. 1.

Comparison of pharyngeal delay time and pharyngeal transit time between groups. With thin-liquid swallowing, the pharyngeal transit (PTT) and pharyngeal delay times (PDT) were significantly longer in the central VCP group $(P=0.003$ and $P=0.016$ by Mann-Whitney test, respectively). The PTT and PDT with thick liquids were also longer in the central VCP group, but the differences were not statistically significant 
Table 1

Details of video fluoroscopic swallowing study parameters for evaluation of the swallowing process

\begin{tabular}{|c|c|}
\hline Parameter of swallowing process & Definition \\
\hline \multicolumn{2}{|l|}{ Oral phase } \\
\hline Lip closure (LC) & Maintaining labial seal to ensure that no food or liquid falls from the mouth \\
\hline Bolus formation $(\mathrm{BF})$ & Mastication and preparation of a semicohesive bolus or ball \\
\hline Oral transit time (OTT) & $\begin{array}{l}\text { The length of time from the first frame showing backward movement of food until the bolus } \\
\text { head reaches the spot where the lower edge of the mandible crosses the tongue base }\end{array}$ \\
\hline Residue of oral cavity (ROC) & Barium residue on the floor of the mouth, tongue, hard palate, and anterolateral sulcus \\
\hline \multicolumn{2}{|l|}{ Pharyngeal phase } \\
\hline Triggering of pharyngeal swallow (TPS) & $\begin{array}{l}\text { Elevation and anterior movement of hyoid and larynx with bolus head passing the spot where } \\
\text { the lower edge of the mandible crosses the tongue base }\end{array}$ \\
\hline $\begin{array}{l}\text { Laryngeal elevation and epiglottic closure } \\
\text { (LEEC) }\end{array}$ & $\begin{array}{l}\text { Getting closer and tilting forward of the arytenoids to the base of epiglottis and closing the } \\
\text { airway as larynx elevates }\end{array}$ \\
\hline $\begin{array}{l}\text { Residue in vallecula and pyriform sinus } \\
\text { (RV \& RP) }\end{array}$ & Barium residue on vallecula and pyriform sinus after swallowing \\
\hline Upper esophageal sphincter release (UESR) & $\begin{array}{l}\text { Opening of upper esophageal sphincter with bolus head reaching the cricopharyngeal } \\
\text { sphincter }\end{array}$ \\
\hline Pharyngeal delayed time (PDT) & $\begin{array}{l}\text { The time from arrival of the bolus at the point where the lower edge of the mandible crosses } \\
\text { the tongue base until the pharyngeal swallow is triggered }\end{array}$ \\
\hline Pharyngeal transit time (PTT) & $\begin{array}{l}\text { The time from the point at which the bolus head passes the spot where the lower edge of the } \\
\text { mandible crosses the tongue base until the bolus tail passes through the cricopharyngeal } \\
\text { region }\end{array}$ \\
\hline Presence of penetration & Presence of the bolus within the laryngeal inlet but above the true vocal cord \\
\hline Presence of aspiration & Passage of the bolus material beyond the level of the true vocal cord \\
\hline
\end{tabular}

The definitions are based on Logemann [3] 
Table 2

\section{Overall incidence of abnormal findings in VFSS}

\begin{tabular}{llll}
\hline Swallowing process & Total no. of patients $(\%)$ & Evaluation parameter & No. of patients (\%) \\
\hline Oral phase abnormalities & $13(46.4 \%)$ & LC & $6(21.4)$ \\
& & BF & $9(32.1)$ \\
Pharyngeal phase abnormalities & $28(100 \%)$ & OTT & $7(25.0)$ \\
& & ROC & $7(25.0)$ \\
& & TPS & $17(60.7)$ \\
& & LEEC & $21(75.0)$ \\
& RV & $21(75.0)$ \\
& & RP & $17(60.7)$ \\
& & UESR & $8(28.6)$ \\
& & Thick/thin PTT & $18(64.3) / 15(53.6)$ \\
& Thick/thin PDT & $13(46.4) / 12(42.8)$ \\
& & Penetration & $20(71.4)$ \\
& & Aspiration & $14(50.0)$ \\
\hline
\end{tabular}

For definitions of abbreviations, see Table 1 
Table 3

Oral phase abnormalities in peripheral and central VCP groups

\begin{tabular}{|c|c|c|c|}
\hline & \multicolumn{2}{|l|}{ Group } & \multirow[t]{2}{*}{$P$ value } \\
\hline & $\begin{array}{r}\text { Peripheral VCP } \\
(n=18)\end{array}$ & $\begin{array}{l}\text { Central VCP } \\
(n=10)\end{array}$ & \\
\hline \multicolumn{4}{|l|}{ LC } \\
\hline Normal & 18 & 4 & $0.001 *$ \\
\hline Abnormal & 0 & 6 & \\
\hline \multicolumn{4}{|l|}{$\mathrm{BF}$} \\
\hline Normal & 15 & 4 & $0.035^{*}$ \\
\hline Abnormal & 3 & 6 & \\
\hline \multicolumn{4}{|l|}{ ROC } \\
\hline Normal & 15 & 6 & 0.207 \\
\hline Abnormal & 3 & 4 & \\
\hline \multicolumn{4}{|l|}{ OTT } \\
\hline Normal & 16 & 5 & 0.063 \\
\hline Abnormal & 2 & 5 & \\
\hline
\end{tabular}

For definitions of abbreviations, see Table 1

${ }^{*} P<0.05$ by Fisher's exact test 
Table 4

Pharyngeal phase abnormalities in peripheral and central VCP groups

\begin{tabular}{|c|c|c|c|}
\hline & \multicolumn{2}{|l|}{ Group } & \multirow[t]{2}{*}{$P$ value } \\
\hline & $\begin{array}{r}\text { Peripheral VCP } \\
(n=18)\end{array}$ & $\begin{array}{l}\text { Central VCP } \\
(n=10)\end{array}$ & \\
\hline \multicolumn{4}{|l|}{ TPS } \\
\hline Normal & 10 & 1 & $0.041^{*}$ \\
\hline Abnormal & 8 & 9 & \\
\hline \multicolumn{4}{|l|}{ LEEC } \\
\hline Normal & 6 & 1 & 0.354 \\
\hline Abnormal & 12 & 9 & \\
\hline \multicolumn{4}{|l|}{ RV } \\
\hline Normal & 5 & 2 & 1.000 \\
\hline Abnormal & 13 & 8 & \\
\hline \multicolumn{4}{|l|}{ RP } \\
\hline Normal & 8 & 3 & 0.689 \\
\hline Abnormal & 10 & 7 & \\
\hline \multicolumn{4}{|l|}{ UESR } \\
\hline Normal & 16 & 4 & $0.011^{*}$ \\
\hline Abnormal & 2 & 6 & \\
\hline \multicolumn{4}{|l|}{ Penetration } \\
\hline$(-)$ & 7 & 1 & 0.194 \\
\hline$(+)$ & 11 & 9 & \\
\hline \multicolumn{4}{|l|}{ Aspiration } \\
\hline$(-)$ & 12 & 2 & $0.046^{*}$ \\
\hline$(+)$ & 6 & 8 & \\
\hline
\end{tabular}

For definitions of abbreviations, see Table 1

${ }^{*} P<0.05$ by Fisher's exact test 